\title{
Neoadjuvant Therapy of Cyclin-Dependent Kinase 4/6 Inhibitors Combined with Endocrine Therapy in HR+/HER2- Breast Cancer: A Systematic Review and Meta-Analysis
}

\author{
Kai Hong ${ }^{a}$ Lingli Yao $^{a}$ Xianneng Sheng ${ }^{b}$ Dan Ye $^{a}$ Yu Guo ${ }^{b}$ \\ ${ }^{a}$ Medicine School, Ningbo University, Ningbo, China; ${ }^{b}$ Department of Thyroid and Breast Surgery, Ningbo City First \\ Hospital, Ningbo, China
}

\section{Keywords}

Hormone receptor+/epidermal growth factor receptor 2breast cancer · Neoadjuvant treatment · Cyclin-dependent kinase 4/6 inhibitors · Endocrine therapy - Neoadjuvant chemotherapy

\footnotetext{
Abstract

Background: Cyclin-dependent kinase (CDK) 4/6 inhibitors have been advocated for adjuvant therapy of metastatic hormone receptor $(\mathrm{HR})+/$ human epidermal growth factor receptor 2 (HER2) - breast cancer (BC). However, the efficiency of adding CDK 4/6 inhibitors to neoadjuvant therapy was not unequivocal. Objective: The aim of the study was to evaluate the efficiency and toxicity of neoadjuvant CDK 4/6 inhibitors + endocrine therapy (ET) versus neoadjuvant endocrine monotherapy or standard neoadjuvant chemotherapy in HR+/HER2- BC. Method: We searched PubMed, the Cochrane Library, Web of Science, and Embase online databases for randomized controlled trials and single-arm studies written in English until April 2021. Results: Five studies comparing CDK 4/6 inhibitors + ET as neoadjuvant treatments to ET alone and 2 studies comparing neoadjuvant CDK 4/6 inhibitors + ET to neoadjuvant chemotherapy were analysed. Neoadjuvant CDK 4/6 inhibitors + ET improved the rate of complete cell cycle arrest (CCCA: central Ki67 < 2.7\%, odds ratio $[\mathrm{OR}]=7.91,95 \%$ confidence interval $[\mathrm{Cl}]=4.81-13.03$, $p<0.001$ ), increased the risk of adverse events (AEs; especially $\geq 3$ AEs; AEs of all grades: $O R=9.10,95 \% \mathrm{Cl}=2.39$ $34.58, p=0.001 ; \mathrm{AEs} \geq 3$ : $\mathrm{OR}=12.24,95 \% \mathrm{Cl}=4.17-35.88$, $p<0.001)$, led to no significant differences in pathological complete response $(p C R)$ in patients with $B C(O R=0.34,95 \%$
}

$\mathrm{Cl}=0.04-2.85, p=0.318$ ) compared to endocrine monotherapy. Moreover, subgroup analysis showed that the 3 types of CDK 4/6 inhibitors all improved the rate of CCCA (ribociclib: $\mathrm{OR}=10.31,95 \% \mathrm{Cl}=3.59-29.61, p<0.001$; palbociclib: $\mathrm{OR}=$ $7.39,95 \% \mathrm{Cl}=1.26-43.40, p=0.027$, and abemaciclib: $\mathrm{OR}=$ $8.28,95 \% \mathrm{Cl}=3.41-20.11, p<0.001)$. Compared to neoadjuvant chemotherapy, neoadjuvant CDK 4/6 inhibitors plus ET decreased the risk of $A E s \geq 3(O R=0.50,95 \% C l=0.29-0.87$, $p=0.015)$ and showed similar ability to reach $\mathrm{pCR}(\mathrm{OR}=0.50$, $95 \% \mathrm{Cl}=0.12-2.07, p=0.342)$ and reduce the residual cancer burden (RCB, RCB 0-1: OR $=0.47,95 \% \mathrm{Cl}=0.18-1.22, p=$ 0.121 ; RCB 2-3: $\mathrm{OR}=2.30,95 \% \mathrm{Cl}=0.89-5.91, p=0.084)$. Conclusions: The results suggested that combination therapy had increased efficacy and toxicity compared to endocrine monotherapy and showed similar efficacy to and better safety than neoadjuvant chemotherapy.

(c) 2021 S. Karger AG, Basel

\section{Introduction}

According to recent statistics, breast cancer (BC) is the most common malignant tumour worldwide and the primary cause of cancer-related death in women [1]. BC is a molecular disease. According to its gene expression, there are 6 molecular subtypes, including luminal A (oestrogen receptor $[\mathrm{ER}]+$, progesterone receptor $[\mathrm{PR}]+$, epidermal growth factor receptor 2 [HER2]-, and Ki67-), luminal $\mathrm{B}(\mathrm{ER}+, \mathrm{PR}+, \mathrm{HER} 2+/-$, and Ki67+), HER2-enriched (HER2+), basal-like subtype (ER-, PR-, and HER2-), normal basal-like, and claudin-low (low expression of cellular adhesion genes) [2-4]. Each BC subtype has dif- 
ferent clinical relevance, treatment strategies, responses to therapy, and long-term survival $[5,6]$. HR+/HER2$\mathrm{BC}$ is the most common subtype, accounting for approximately $65 \%$ of all BCs [7]. CDK $4 / 6$ inhibitor is frequently used for patients with metastatic HR+/HER2- BC, which can also be combined with endocrine therapy (ET) to improve the prognosis of patients with metastatic disease. The CDKs are a large family of serine-threonine kinases, which have important roles in cell cycle regulation [8]. Cyclin D binds CDK 4/6 and then hyper-phosphorylates retinoblastoma protein, which results in cancer cell cycle progression from the G1 phase to the $S$ phase. CDK 4/6 inhibitors can block the hyper-phosphorylation of phosphorylates retinoblastoma protein, causing G1 arrest and hindering proliferation $[9,10]$. Given the antiproliferative effects of CDK 4/6 inhibitors, Ki67 levels are used to estimate the efficacy of combining CDK 4/6 inhibitors with ET in present studies [11].

Neoadjuvant therapy is of great value to patients with early BC. It promotes breast-conserving surgery and can be useful to evaluate tumour biomarkers to confirm a proof-of-principle activity and distinguish patients who are responsive or resistant to therapy $[12,13]$. Achieving pCR in HR + BC through chemotherapy is more difficult than in other subtypes [14]. Previously, CDK 4/6 inhibitors were always combined with ET for metastatic $\mathrm{BC}$ as a rescue treatment for patients with advanced disease. To further improve the efficacy of neoadjuvant therapy, CDK 4/6 inhibitors were combined with ET as a neoadjuvant therapeutic regimen. A randomized phase II trial [15] indicated that ribociclib combined with letrozole significantly improved the number of patients who achieved CCCA versus letrozole alone in postmenopausal women with a new diagnosis of surgically resectable, grade II/III HR+/HER2- BC. Another phase II randomized multicentre trial (PALLET) [14] showed that adding palbociclib to letrozole improved the number of patients with CCCA compared to using letrozole alone in postmenopausal women with unilateral, operable, non-metastatic, HR+/HER2- tumours that were $\geq 2 \mathrm{~cm}$. In addition, a non-randomized, singlearm phase II trial (NeoPalAna) [16] evaluated the antiproliferation effects of palbociclib in stage II/III ER+/ HER2- BC. Although patients were non-randomly assigned to 3 groups according to their PIK3CA status and level of endocrine resistance, it showed the CCCA rate of overall patients before and after adding palbociclib to anastrozole. Furthermore, a randomized, multicentre, open-label, phase II study (neoMONARCH) [17] found that more patients achieved CCCA in abemaciclib-containing arms than in anastrozole alone arms. Recently, a randomized, phase II study (FELINE) [18], suggested that, among postmenopausal women with tumours $>2$ $\mathrm{cm}$ or node + ER+/HER2 - BC, ribociclib + letrozole achieved a higher rate of CCCA than letrozole + placebo. Moreover, 2 studies (NeoPAL and CORALLEEN) [19, 20] that included experimental groups who received neoadjuvant CDK 4/6 inhibitors + ET and control groups who received a standard chemotherapy regimen, showed similar effectiveness and better safety of CDK $4 / 6$ inhibitors + ET than chemotherapy. We designed the current meta-analysis to further clarify the benefits and toxicities of the use of CDK 4/6 inhibitors + ET in $\mathrm{HR}+/ \mathrm{HER} 2-\mathrm{BC}$ and to explore any differences among 3 types of CDK 4/6 inhibitors (ribociclib, palbociclib, and abemaciclib).

\section{Methods}

Search Strategy

We searched PubMed (70), the Cochrane Library (135), Web of Science (158), and Embase (169) online databases to identify articles on neoadjuvant therapy with CDK 4/6 inhibitors combined with ET until April 18, 2021. The search was restricted to English, using the following keywords: "Neoadjuvant," "breast OR mammary," "cancer OR tumour OR neoplasm OR adenocarcinoma OR carcinoma," "CDK 4/6 inhibitor* OR Palbociclib OR Abemaciclib OR Ribociclib OR Cyclin-dependent kinase 4/6 inhibitor*." Reference lists were reviewed and checked for other relevant studies. For unpublished trials, we contacted the corresponding authors through the ClinicalTrials.gov website to obtain the relevant data. Two authors checked selected articles gained from the search. The 2 reviewers discussed and resolved disagreements, consulting the third author when necessary. A PRISMA flow diagram was devised to record article selection.

\section{Study Selection}

Two authors independently conducted the selection. The inclusion criteria were as follows: (1) randomized trials (besides the NeoPalAna trial); (2) patients diagnosed with HR+/HER2- BC; (3) experimental arms patients received neoadjuvant CDK 4/6 inhibitors + ET; and (4) complete data and published in English.

The exclusion criteria were as follows: (1) studies with incomplete or missing information; (2) retrospective or prospective observational cohort studies; (3) single-arm clinical trials without control data; and (4) reviews, case reports, and editorials. For duplicate published trials, the complete trial was chosen.

\section{Data Extraction and Quality Assessment}

We extracted the following information from eligible studies: (1) the name of the first author; (2) year of publication; (3) countries and characteristics of participants; (4) interventions of the experiment and control arms; and (5) primary and secondary outcomes. The risk of bias of the included studies was assessed using the Cochrane Collaboration tool by Review Manager 5.4 software, which, as shown in Figure 1, contains sequence generation, allocation concealment, blinding, incomplete outcome data, selective reporting, and other bias. Two authors carried out data extraction and quality assessment, and they resolved disagreements through discussion.

Data Synthesis and Analysis

All statistical analyses were performed using Stata 16.0. The outcomes of this meta-analysis included CCCA, AEs, pCR, and residual cancer burden (RCB). 


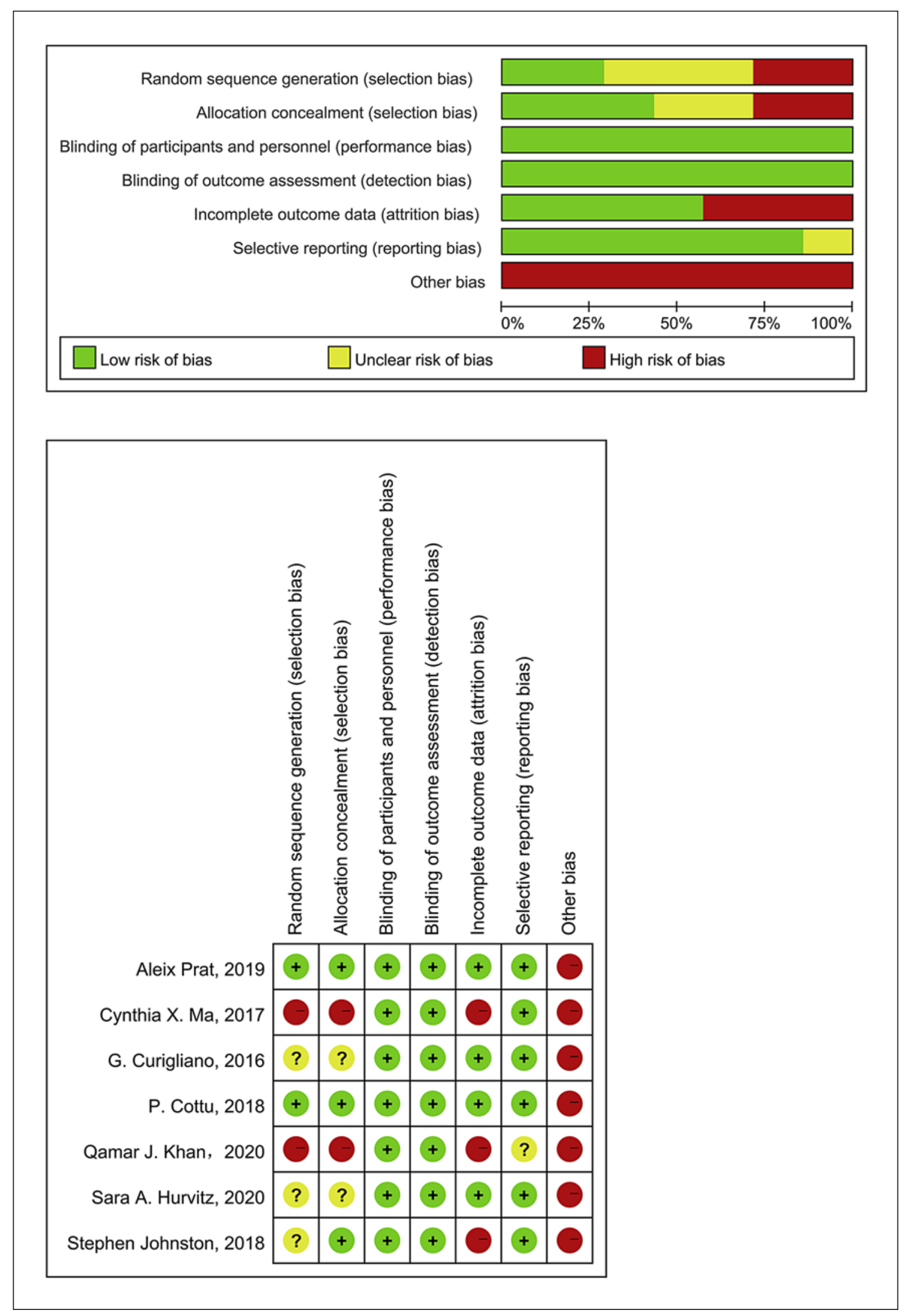

Fig. 1. Table and graphs of the risk of bias for the included studies. 


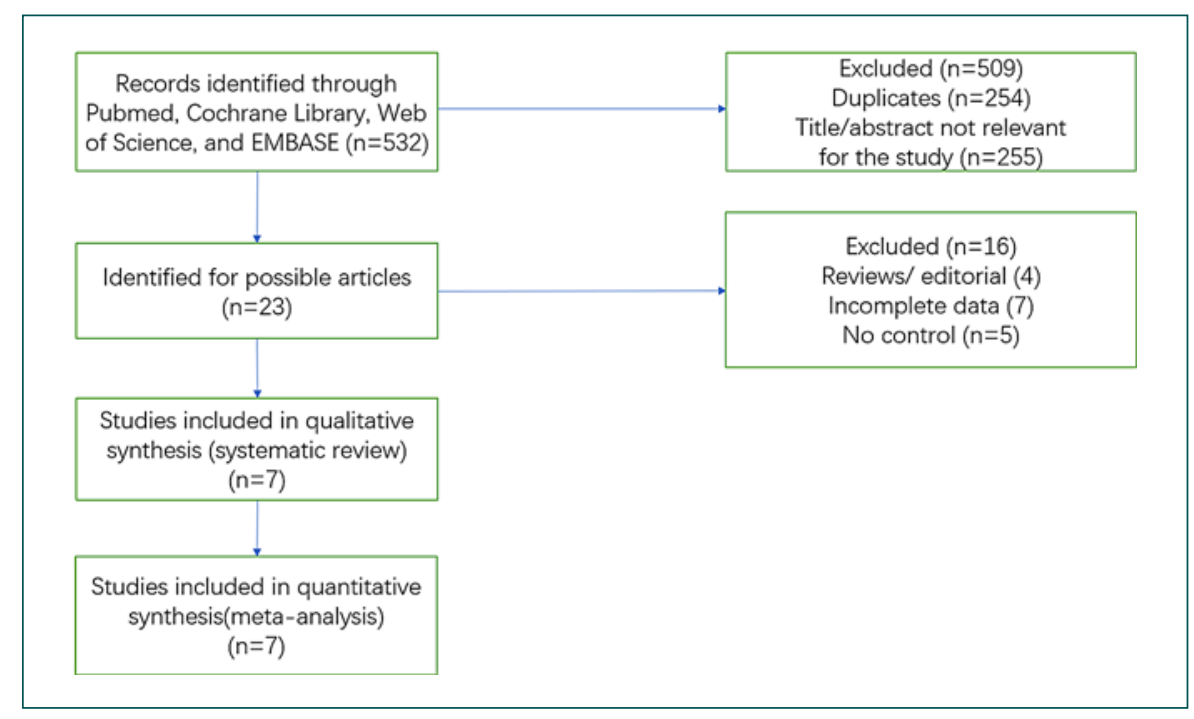

Fig. 2. PRISMA flow diagram.

The pooled odds ratio (OR) with $95 \%$ confidence interval (CI) was calculated for dichotomous variables. Statistical heterogeneity was tested using the $I^{2}$ index. For $I^{2}<50 \%$ and $p>0.05$, the fixedeffect model was applied, and if $I^{2} \geq 50 \%$ or $p<0.05$ indicated obvious heterogeneity, we aimed to identify the sources of heterogeneity. If the sources were not found, the random-effects model was used. Sensitivity analysis was used to prove the stability of our results.

\section{Results}

\section{Search Results}

Figure 2 displays the selection process. After exclusion of 254 duplicates, 255 studies were excluded after a review of the titles and abstracts. We then excluded 16 articles, including reviews and editorials $(n=4)$, due to incomplete data $(n=7)$ and no control $(n=5)$. Finally, 7 articles met the inclusion criteria.

\section{Study Characteristics}

Table 1 shows the basic characteristics of the included studies and patients. Table 2 shows the outcomes of the 7 trials. Five of the 7 trials investigated CDK $4 / 6$ inhibitors + ET compared to endocrine monotherapy. Among these trials, we included a single-arm trial that still met the inclusion criteria because it compared the index after patients received anastrozole for 4 weeks followed by addition of palbociclib. All 5 trials reported the CCCA at week 2 ; among them, 3 trials reported the grade $3 / 4$ AEs of patients of each group, 2 trials only reported the grade $3 / 4$ AEs of the whole patients, we did not record the data of these 2 trials. Two trials reported the pCR in BC.

Two of the 7 trials were on CDK $4 / 6$ inhibitors + ET versus chemotherapy. Both trials reported the grade $3 / 4$ AEs of each group, $\mathrm{pCR}$ of $\mathrm{BC}$, and rate of the RCB $0-1$.

\section{CCCA Analysis}

Five trials enrolling 482 patients were included in the final analysis for CCCA (Fig. 3). The CCCA of all patients was first analysed using the fixed-effects model as no obvious heterogeneity was found $\left(I^{2}=33.8 \%, p=0.196\right)$ in the data. The results indicated that using neoadjuvant CDK 4/6 inhibitors + ET achieved a higher CCCA rate than using neoadjuvant endocrine monotherapy $(\mathrm{OR}=$ $7.91,95 \% \mathrm{CI}=4.81-13.03, p<0.001)$. Then we, respectively, analysed the CCCA of ribociclib, palbociclib, and abemaciclib (Fig. 3). The random-effects model was used given the existence of heterogeneities in the palbociclib subgroup $\left(I^{2}=82.4 \%, p=0.017\right)$. The results showed that adding any of the CDK 4/6 inhibitors to ET as neoadjuvant treatment led to a significantly higher rate of CCCA $(\mathrm{OR}=10.31,95 \% \mathrm{CI}=3.59-29.61, p<0.001 ; \mathrm{OR}=7.39$, $95 \% \mathrm{CI}=1.26-43.40, p=0.027 ; \mathrm{OR}=8.28,95 \% \mathrm{CI}=$ $3.41-20.11, p<0.001$, respectively).

\section{AE Analysis}

In the trials of CDK 4/6 inhibitors + ET, 2 trials with 315 patients, reported AEs of all grades, and 3 trials with 435 patients reported AEs $\geq 3$. The fixed-effects model was used because no heterogeneity was found $\left(I^{2}=0, p=\right.$ $0.827 ; I^{2}=0, p=0.464$, respectively) (Fig. 3 ). When analysing the trials comparing CDK 4/6 inhibitors with chemotherapy, the fixed-effects model was used given the absence of heterogeneity $\left(I^{2}=0, p=0.591\right)$. The results indicated that CDK 4/6 inhibitors clearly increased the risk of AEs when combined with ET, especially AEs $\geq 3$ $(\mathrm{OR}=9.10,95 \% \mathrm{CI}=2.39-34.58, p=0.001 ; \mathrm{OR}=12.24$, $95 \% \mathrm{CI}=4.17-35.88, p<0.001$, respectively) (Fig. 3). Further, the CDK $4 / 6$ inhibitors + ET versus chemotherapy trials demonstrated a decrease in the risk of AEs $\geq 3$ (OR $=0.50,95 \% \mathrm{CI}=0.29-0.87, p=0.015)$. 


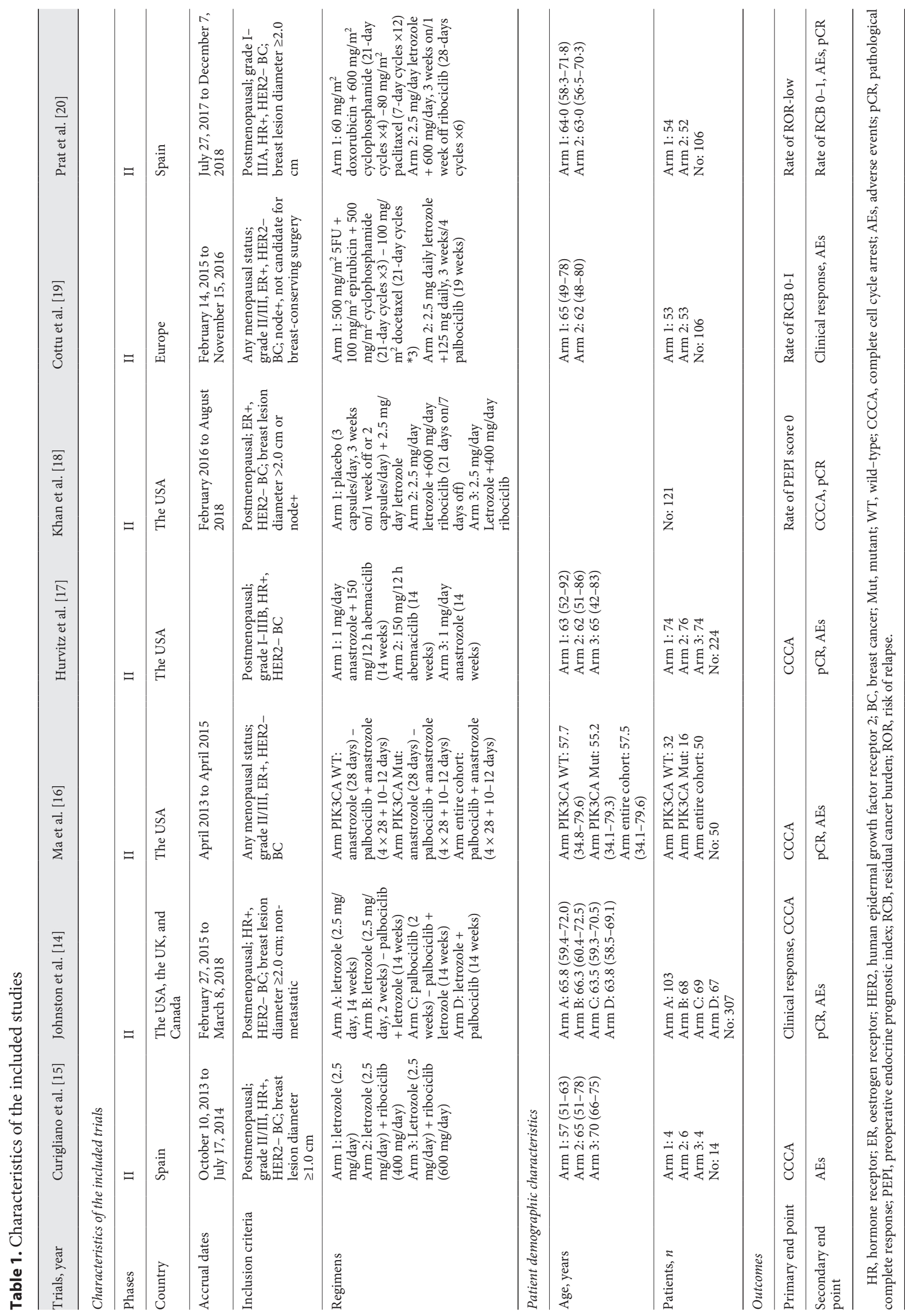




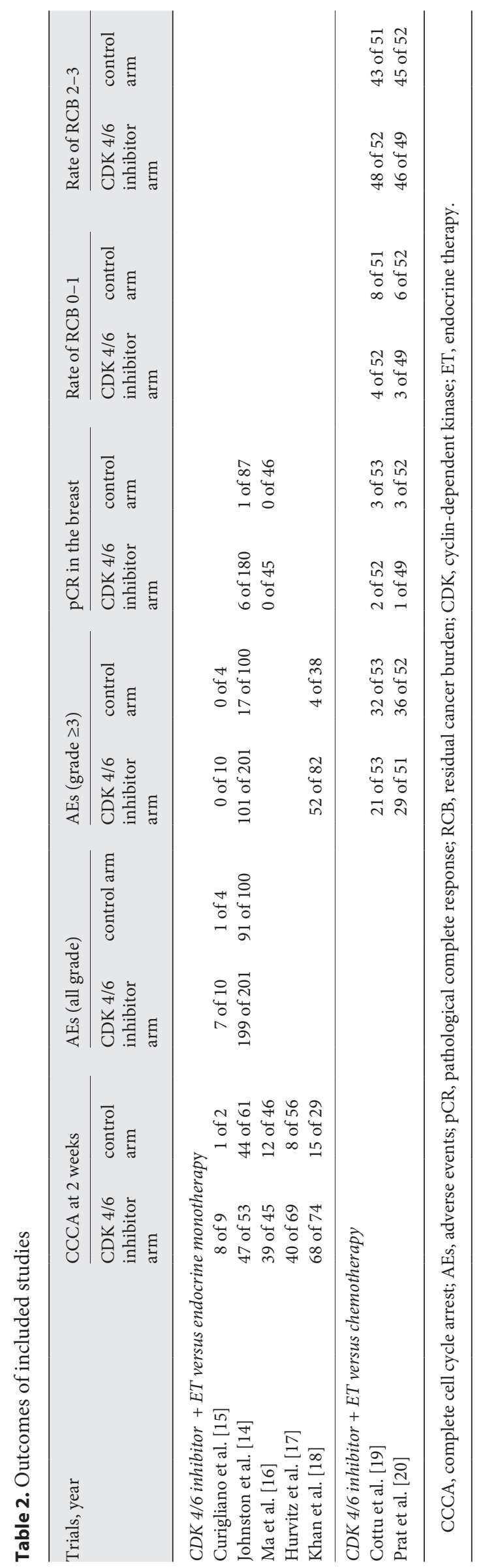

\section{pCR in the Analysis of $B C$}

As depicted in Figure 4, the results from 4 trials reported the rate of $\mathrm{pCR}$ of $\mathrm{BC}$, with no significant differences between treatment with CDK 4/6 inhibitors + ET versus endocrine monotherapy or chemotherapy $(\mathrm{OR}=0.34,95 \% \mathrm{CI}$ $=0.04-2.85, p=0.318 ; \mathrm{OR}=0.50,95 \% \mathrm{CI}=0.12-2.07, p=$ 0.342 , respectively); the fixed-effects model was used because no heterogeneity was found $\left(I^{2}=0, p=0.827\right)$.

\section{Rate of RCB Analysis}

The data regarding the rate of $\mathrm{RCB}$ in the 2 trials of CDK 4/6 inhibitors + ET versus chemotherapy indicated that the rate of RCB $0-1$ in the CDK 4/6 inhibitors + ET groups was lower than that in chemotherapy groups (OR $=0.47,95 \% \mathrm{CI}=0.18-1.22, p=0.121$ ) (Fig. 4). In contrast, the rate of the RCB 2-3 was higher in the CDK 4/6 inhibitors + ET groups $(\mathrm{OR}=2.30,95 \% \mathrm{CI}=0.89-5.91, p$ $=0.084$ ). Regarding the effect of reducing cancer burden, neoadjuvant chemotherapy was better than neoadjuvant CDK 4/6 inhibitors + ET, although there were no significant differences in the data. The fixed-effects model was used due to the lack of heterogeneity $\left(I^{2}=0, p=0.911 ; I^{2}\right.$ $=0, p=946$, respectively).

\section{Sensitivity Analysis of the Primary Outcome and Publication Bias}

The sensitivity analysis showed that the 5 included studies had no conspicuous alterations of the primary outcome, and only the study by Stephen Johnston demonstrated heterogeneity (Fig. 5). The sensitivity analysis indicated that the results of CCCA in the patients of these studies were relatively reliable and stable. It was not necessary to assess the publication bias because the number of studies is less than 10 .

\section{Heterogeneity Analysis}

The results of sensitivity and subgroup analyses indicated that heterogeneities existed in the PALLET and NeoPalAna trials. In the NeoPalAna trial [16], patients first received 4 weeks anastrozole, followed by palbociclib; the study compared 4 weeks anastrozole monotherapy with 6 weeks anastrozole +2 weeks palbociclib treatment, which differed from the regimen used in other studies. This may be the source of heterogeneity. However, due to the lack of a more detailed subgroup analysis, the exact source of heterogeneity in these trials could not be determined. No obvious heterogeneities were detected in other studies.

\section{Discussion/Conclusion}

At present, neoadjuvant chemotherapy and ET are the most commonly used neoadjuvant treatment for patients with HR+/HER2- BC. Neoadjuvant treatment is valuable 


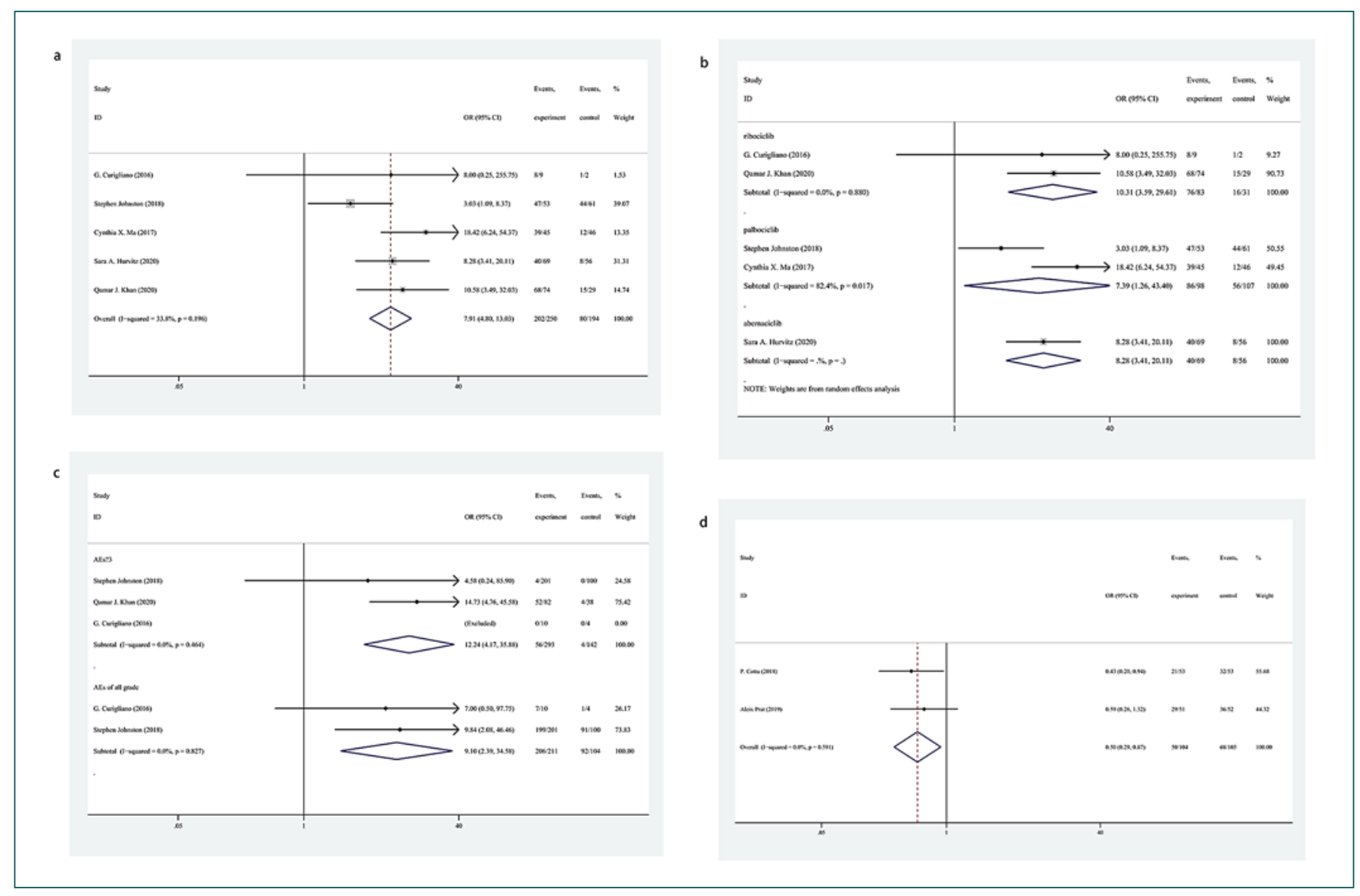

Fig. 3. a Forest plot of CCCA of CDK 4/6 inhibitors + ET versus endocrine monotherapy. b Forest plot of subgroup analysis on CCCA of CDK 4/6 inhibitors + ET versus endocrine monotherapy. c Forest plot of AEs of CDK 4/6 inhibitors + ET versus endocrine monotherapy. d Forest plot of AEs of CDK 4/6 inhibitors + ET versus chemotherapy. CCCA, complete cell cycle arrest; CDK, cyclin-dependent kinase; ET, endocrine therapy; AEs, adverse events; $\mathrm{OR}$, odds ratio; $\mathrm{CI}$, confidence interval.

to patients who wish to achieve breast-conserving surgery, those with tumours that are too large to receive surgery, and can predict the resistant and responsive subgroups of patients [21-23]. Although the effect of neoadjuvant chemotherapy had been confirmed [24], the toxicity and resistance of chemotherapeutic drugs have halted the progress of neoadjuvant treatment. Adjuvant ET after surgery is a relatively safe regimen that is effective for patients with $\mathrm{HR}+\mathrm{BC}$, and patients with luminal $\mathrm{A}$ and luminal $\mathrm{B}$ type $\mathrm{BC}$ are usually recommended to receive ET for 5 years [25]. In addition, many studies have discussed the effectiveness of neoadjuvant ET strategies [26-28]. Indeed, a previous meta-analysis reported that neoadjuvant ET, as a monotherapy, showed similar efficacy to neoadjuvant chemotherapy but with significantly better safety [29]. Nevertheless, similar to chemotherapy, endocrine resistance, and effectiveness of ET remain key concerns for these patients. Data have demonstrated that the cyclin D-CDK 4/6-INK4-Rb pathway remains active in endocrine-resistant cancer cells, and the addition of CDK $4 / 6$ inhibitors may solve this problem [30, 31].
However, CDK 4/6 inhibitors are relatively new drugs for HR+/HER2- BC and are not commonly used for neoadjuvant treatment. Few studies have examined neoadjuvant CDK 4/6 inhibitor therapy, although a previous case report showed that CDK 4/6 inhibitors were effective as a neoadjuvant treatment [31]. Our study is the first metaanalysis to evaluate the effects and safety of neoadjuvant CDK 4/6 inhibitor + ET versus endocrine monotherapy or chemotherapy for HR+, HER2- BC, and discussed the effectiveness of 3 types of CDK 4/6 inhibitors separately.

The use of neoadjuvant CDK 4/6 inhibitors represents an important future research direction. The results of our study indicated that compared to endocrine monotherapy, patients who received combination therapy attained a higher rate of CCCA, although they did not obtain higher pCR and had more AEs of all grades, especially grades $\geq 3$. Compared to neoadjuvant chemotherapy, the ability to decrease the RCB of combination therapy was slightly worse; however, as the results lacked statistical significance, the combination therapy and chemotherapy regimen might have similar effectiveness, although this 
Fig. 4. a Forest plot of $\mathrm{pCR}$ in the breast with CDK 4/6 inhibitors + ET versus endocrine monotherapy and chemotherapy. b Forest plot of the rate of RCB with CDK $4 / 6$ inhibitors + ET versus chemotherapy. pCR, pathological complete response; CDK, cyclin-dependent kinase; RCB, residual cancer burden; ET, endocrine therapy; OR, odds ratio; $\mathrm{CI}$, confidence interval.
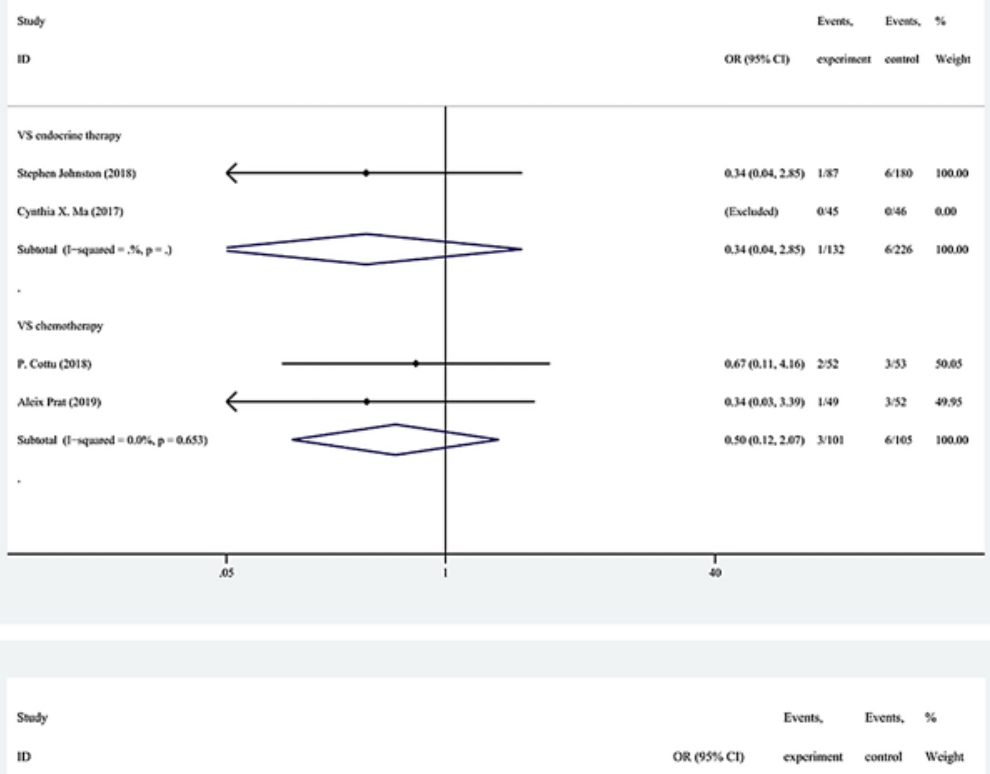

RCB 0-1

P. Cestu (2018)

Aleix Prat (2019)

Subsocal (1-squered $-0.0 \%, p-0.911)$

RCB 2-3

P. Cestu (2018)

Akeix Prat (2019)

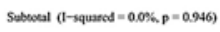

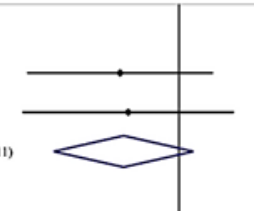

a.45(0.13, 1.59) $\quad 452 \quad 8551 \quad 57.70$

aso(0.12,2.12) 3/49 6/52 4230

$0.47(0.18,1.22) \quad 7101 \quad 147103 \quad 100.00$

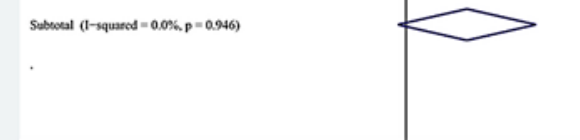

.05

$223(0.63,7.94) \quad 4852 \quad 43 / 51 \quad 35.54$

$239(0.58,9.90) \quad 46.49 \quad 45 / 52 \quad 44.46$

$230(0.59) .591) \quad 94701 \quad 85 / 103 \quad 100000$

Fig. 5. Sensitivity analysis.

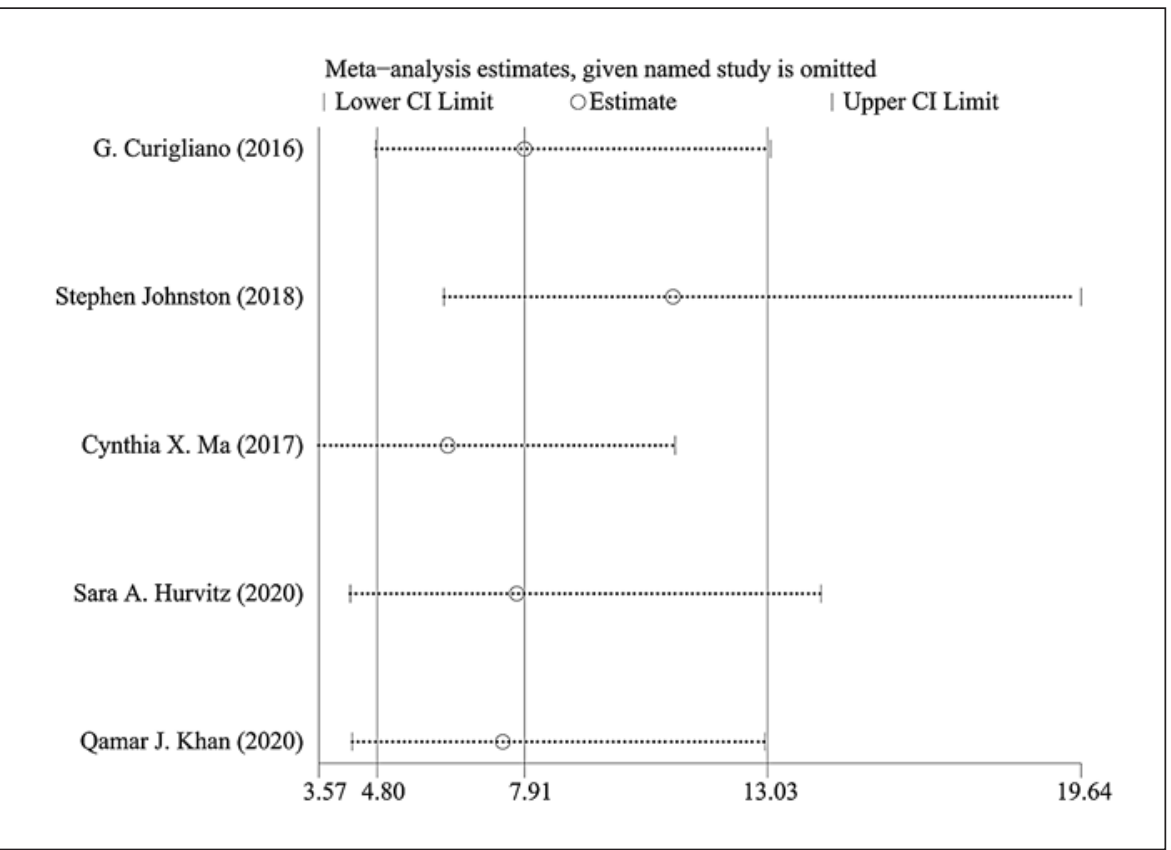


should be confirmed through further studies. The safety of CDK 4/6 inhibitors + ET was significantly better than chemotherapy. Besides, subgroup analysis showed that ribociclib, palbociclib, and abemaciclib all had positive effects when added to the ET as a neoadjuvant treatment.

In the PALLET trial [14], CCCA at 2 weeks was higher in the palbociclib + letrozole group (47 [89\%] of 53 patients; 95\% CI, 76\%-96\%) than in the palbociclib alone group (44 [72\%] of 61 patients; 95\% CI, 59\%-82\%; $p=$ $0.04)$. Moreover, at the end of treatment, CCCA was observed in 38 of 65 (58.5\%) patients in the letrozole group compared to 113 of $125(90.4 \%)$ in the palbociclib + letrozole groups (OR, 6.83; 95\% CI, 3.12-14.98; $p=0.001$ ); this suggests palbociclib is effective in cases of endocrine resistance. Another noteworthy finding is that the addition of palbociclib decreased the ratios of some AEs, including hot flush, arthralgia, headache, breast pain, hypertension, depression, pain in extremity, and myalgia. However, as this finding was not discussed fully, we hope future studies will cover it in greater detail.

Previous studies have demonstrated that $\mathrm{PCR}$ is more difficult to achieve in luminal-type $\mathrm{BC}$ than in other types [32-34]. As patients receiving neoadjuvant ET rarely achieve pCR $[35,36]$, more treatment strategies must be considered to enhance neoadjuvant treatment in luminal-type patients. However, in the PALLET, NeoPalAna, NeoPAL, and CORALLEEN trials, all of which reported pCR, showed no significant differences between neoadjuvant ET alone, neoadjuvant CDK 4/6 inhibitors + ET or neoadjuvant chemotherapy $[14,16,19,20]$. This may be because ER + tumours are slower growing and need a longer time to react to the predominantly anti-proliferative treatments [37].

At present, the main problem with the use of CDK $4 / 6$ inhibitors is the lack of accurate biomarkers beyond ER status. Indeed, some luminal patients show a good response to CDK 4/6 inhibitors, while others do not; therefore, relying on ER status alone is insufficient to decide on CDK 4/6 inhibitors as a therapeutic regimen.

PIK3CA mutations are common in ER + BC $[38,39]$. To further explore this question, patients were divided into PIK3CA WT, or Mut cohorts according to their PIK3CA status in the NeoPalAna trial. The results demonstrated that PIK3CA WT tumours had greater variability in Ki67 response and were more responsive to palbociclib. As a result, the authors stated that CDK 4/6 inhibitors should not be selected on the basis of the PIK3CA status [16]. This viewpoint was shared by the researchers involved in the PALOMA-3 trial [40], in which PIK3CA status in circulating tumour DNA did not indicate a benefit of palbociclib treatment. Moreover, to seek out potential clinical and molecular responsive markers for palbociclib, Ma et al. [16] discussed whether patients' menopausal status influenced the CCCA, tumours histology and grade, PgR status, and mutations identified in the 83 -gene panel NGS. The results demonstrated that palbociclib benefits were observed in all subgroups. In the PALOMA-2 trial [41], ER, Rb, p16, cyclin D1, and Ki67 failed to indicate a benefit of adding palbociclib to ET. Although the explorations of potential predictive markers for CDK 4/6 inhibitors are underway, the expected discovery has not yet appeared.

There are several limitations of our analysis. First, there are few studies of neoadjuvant CDK 4/6 inhibitors, although some trials are ongoing, which may further validate our findings. Second, the baseline of the included patients was not strictly controlled; any menopausal status of patients was included in the 2 trials, and patients with grade I tumours were included in 2 studies. Third, no data of long-term survival were reported from the included trials. Although the Ki67 levels are associated with risks of tumour relapse and benefits from therapy [4244], uncertainties of whether a higher rate of CCCA is associated with improved long-term outcome should be verified in studies with further follow-up. Finally, given the abundance of CDK 4/6 inhibitors, endocrine drugs, and chemotherapy regimens, we were unable to acquire sufficient data for each type of drug.

Our meta-analysis showed that adding neo-CDK 4/6 inhibitors to neoadjuvant ET led to a higher rate of CCCA but a greater incidence of AEs versus endocrine monotherapy and showed similar efficacy and a reduced incidence of AEs versus neoadjuvant chemotherapy. The 3 treatments investigated showed no significant differences in pCR. Further studies are required to explore whether using neoadjuvant CDK 4/6 inhibitors can significantly increase the long-term survival of patients with luminal $\mathrm{BC}$ and which groups of luminal BC will most benefit from neoadjuvant CDK 4/6 inhibitor treatment.

\section{Acknowledgments}

We wish to thank all of the patients with $\mathrm{BC}$ who participated in this study.

\section{Statement of Ethics}

An ethics statement is not applicable because this study is based exclusively on published literature.

\section{Conflict of Interest Statement}

The authors have no conflicts of interest to declare. 


\section{Funding Sources}

The article has no sources of funding.

\section{Data Availability Statement}

The data adopted in this meta-analysis are available from the corresponding author on reasonable request.

\section{Author Contributions}

K.H., L.Y., X.S., D.Y., and Y.G. performed the statistical analysis and wrote the manuscript. K.H., L.Y., and D.Y. conceived the study design and participated in the manuscript writing. X.S and Y.G. revised and edited the manuscript. All authors read the final manuscript.

\section{References}

1 Wu H-J, Chu P-Y. Recent discoveries of macromolecule- and cell-based biomarkers and therapeutic implications in breast cancer. Int J Mol Sci. 2021 Jan;22(2):636.

2 Perou CM, Sørlie T, Eisen MB, van de Rijn M, Jeffrey SS, Rees CA, et al. Molecular portraits of human breast tumours. Nature. 2000 Aug; 406(6797):747-52.

3 Prat A, Parker JS, Karginova O, Fan C, Livasy C, Herschkowitz JI, et al. Phenotypic and molecular characterization of the claudin-low intrinsic subtype of breast cancer. Breast Cancer Res. 2010 Oct;12(5):R68.

4 Garrido-Castro AC, Lin NU, Polyak K. Insights into molecular classifications of triplenegative breast cancer: improving patient selection for treatment. Cancer Discov. 2019 Feb;9(2):176-98.

5 Sørlie T, Perou CM, Tibshirani R, Aas T, Geisler S, Johnsen H, et al. Gene expression patterns of breast carcinomas distinguish tumor subclasses with clinical implications. Proc Natl Acad Sci U S A. 2001 Sep;98(19): 10869-74.

6 Sotiriou C, Neo SY, McShane LM, Korn EL, Long PM, Jazaeri A, et al. Breast cancer classification and prognosis based on gene expression profiles from a population-based study. Proc Natl Acad Sci U S A. 2003;100(18): 10393-8.

7 Lindström LS, Karlsson E, Wilking UM, Johansson U, Hartman J, Lidbrink EK, et al. Clinically used breast cancer markers such as estrogen receptor, progesterone receptor, and human epidermal growth factor receptor 2 are unstable throughout tumor progression. J Clin Oncol. 2012 Jul;30(21):2601-8.

8 Finn RS, Aleshin A, Slamon DJ. Targeting the cyclin-dependent kinases (CDK) 4/6 in estrogen receptor-positive breast cancers. Breast Cancer Res. 2016 Feb;18(1):17.

9 VanArsdale T, Boshoff C, Arndt KT, Abraham RT. Molecular pathways: targeting the cyclin D-CDK4/6 axis for cancer treatment. Clin Cancer Res. 2015 Jul;21(13):2905-10.

10 Lin Z, Zhang X, Zhao F, Ru S. Bisphenol S promotes the cell cycle progression and cell proliferation through ER $\alpha$-cyclin D-CDK4/6$\mathrm{pRb}$ pathway in MCF-7 breast cancer cells. Toxicol Appl Pharmacol. 2019 Mar;366:7582 .
11 Arnedos M, Bayar MA, Cheaib B, Scott V, Bouakka I, Valent A, et al. Modulation of Rb phosphorylation and antiproliferative response to palbociclib: the preoperative-palbociclib (POP) randomized clinical trial. Ann Oncol. 2018 Aug;29(8):1755-62.

12 Wolmark N, Wang J, Mamounas E, Bryant J, Fisher B. Preoperative chemotherapy in patients with operable breast cancer: nine-year results from National Surgical Adjuvant Breast and Bowel Project B-18. J Natl Cancer Inst Monogr. $2001 \mathrm{Dec}(30): 96-102$.

13 Kaufmann M, von Minckwitz G, Smith R, Valero V, Gianni L, Eiermann W, et al. International expert panel on the use of primary (preoperative) systemic treatment of operable breast cancer: review and recommendations. J Clin Oncol. 2003 Jul;21(13):2600-8.

14 Johnston S, Puhalla S, Wheatley D, Ring A, Barry P, Holcombe C, et al. Randomized phase II study evaluating palbociclib in addition to letrozole as neoadjuvant therapy in estrogen receptor-positive early breast cancer: PALLET trial. J Clin Oncol. 2019 Jan;37(3): 178-89.

15 Curigliano G, Gómez Pardo P, Meric-Bernstam F, Conte P, Lolkema MP, Beck JT, et al. Ribociclib plus letrozole in early breast cancer: a presurgical, window-of-opportunity study. Breast. 2016 Aug;28:191-8

16 Ma CX, Gao F, Luo J, Northfelt DW, Goetz M, Forero A, et al. NeoPalAna: neoadjuvant palbociclib, a cyclin-dependent kinase 4/6 inhibitor, and anastrozole for clinical stage 2 or 3 estrogen receptor-positive breast cancer. Clin Cancer Res. 2017 Aug;23(15):4055-65.

17 Hurvitz SA, Martin M, Press MF, Chan D, Fernandez-Abad M, Petru E, et al. Potent cellcycle inhibition and upregulation of immune response with abemaciclib and anastrozole in neoMONARCH, phase II neoadjuvant study in HR+/HER2(-) breast cancer. Clin Cancer Res. 2020 Feb;26(3):566-80.

18 Khan QJ, O’Dea A, Bardia A, Kalinsky K, Wisinski KB, O'Regan R, et al. Letrozole + ribociclib versus letrozole + placebo as neoadjuvant therapy for ER+ breast cancer (FELINE trial). J Clin Oncol. 2020;38(15 suppl):505.

19 Cottu P, D'Hondt V, Dureau S, Lerebours F, Desmoulins I, Heudel PE, et al. Letrozole and palbociclib versus chemotherapy as neoadjuvant therapy of high-risk luminal breast cancer. Ann Oncol. 2018 Dec;29(12):2334-40.
20 Prat A, Saura C, Pascual T, Hernando C, Munoz M, Pare L, et al. Ribociclib plus letrozole versus chemotherapy for postmenopausal women with hormone receptor-positive, HER2-negative, luminal B breast cancer (CORALLEEN): an open-label, multicentre, randomised, phase 2 trial. Lancet Oncol. 2020 Jan;21(1):33-43.

21 Smith IE, Dowsett M, Ebbs SR, Dixon JM, Skene A, Blohmer JU, et al. Neoadjuvant treatment of postmenopausal breast cancer with anastrozole, tamoxifen, or both in combination: the Immediate Preoperative Anastrozole, Tamoxifen, or Combined with Tamoxifen (IMPACT) multicenter doubleblind randomized trial. J Clin Oncol. 2005 Aug;23(22):5108-16.

22 Abrial C, Mouret-Reynier MA, Curé H, Feillel V, Leheurteur M, Lemery S, et al. Neoadjuvant endocrine therapy in breast cancer. Breast. 2006 Feb;15(1):9.

23 Chaudhary LN, Wilkinson KH, Kong A. Triple-negative breast cancer: who should receive neoadjuvant chemotherapy? Surg Oncol Clin N Am. 2018 Jan;27(1):141-53.

24 Dubsky PC, Singer CF, Egle D, Wette V, Petru E, Balic M, et al. The EndoPredict score predicts response to neoadjuvant chemotherapy and neoendocrine therapy in hormone receptor-positive, human epidermal growth factor receptor 2-negative breast cancer patients from the ABCSG-34 trial. Eur J Cancer. 2020 Jul;134:99-106

25 Francis PA, Pagani O, Fleming GF, Walley BA, Colleoni M, Láng I, et al. Tailoring adjuvant endocrine therapy for premenopausal breast cancer. N Engl J Med. 2018 Jul;379(2): 122-37.

26 Fontein DB, Charehbili A, Nortier JW, Meershoek-Klein Kranenbarg E, Kroep JR, Putter $\mathrm{H}$, et al. Efficacy of six month neoadjuvant endocrine therapy in postmenopausal, hormone receptor-positive breast cancer patients: a phase II trial. Eur J Cancer. 2014 Sep;50(13): 2190-200.

27 Liang X, Briaux A, Becette V, Benoist C, Boulai A, Chemlali W, et al. Molecular profiling of hormone receptor-positive, HER2-negative breast cancers from patients treated with neoadjuvant endocrine therapy in the CARMINA 02 trial (UCBG-0609). J Hematol Oncol. 2018 Oct;11(1):124. 
28 Kim HJ, Noh WC, Lee ES, Jung YS, Kim LS, Han W, et al. Efficacy of neoadjuvant endocrine therapy compared with neoadjuvant chemotherapy in pre-menopausal patients with oestrogen receptor-positive and HER2-negative, lymph node-positive breast cancer. Breast Cancer Res. 2020 May;22(1): 54.

29 Spring LM, Gupta A, Reynolds KL, Gadd MA, Ellisen LW, Isakoff SJ, et al. Neoadjuvant endocrine therapy for estrogen receptor-positive breast cancer: a systematic review and meta-analysis. JAMA Oncol. 2016 Nov;2(11): 1477-86.

30 Pandey K, An HJ, Kim SK, Lee SA, Kim S, Lim SM, et al. Molecular mechanisms of resistance to CDK4/6 inhibitors in breast cancer: a review. Int J Cancer. 2019 Sep;145(5):1179-88.

31 Jung SU, Jung M, Choi JH, Jeon CW. Palbociclib with letrozole as second-line neo-systemic therapy after failure of neo-adjuvant chemotherapy for luminal type breast cancer: a case report. Medicine. 2021 Apr;100(14): e25175.

32 Esserman LJ, Berry DA, Cheang MC, Yau C, Perou CM, Carey L, et al. Chemotherapy response and recurrence-free survival in neoadjuvant breast cancer depends on biomarker profiles: results from the I-SPY 1 TRIAL (CALGB 150007/150012; ACRIN 6657) Breast Cancer Res Treat. 2012 Apr;132(3): 1049-62.
33 Huang L, Xu AM. Short-term outcomes of neoadjuvant hormonal therapy versus neoadjuvant chemotherapy in breast cancer: systematic review and meta-analysis of randomized controlled trials. Expert Rev Anticancer Ther. 2017 Apr;17(4):327-34.

34 LeVasseur N, Willemsma KA, Li H, Gondara L, Yip WC, Illmann C, et al. Efficacy of neoadjuvant endocrine therapy versus neoadjuvant chemotherapy in ER-positive breast cancer: results from a prospective institutional database. Clin Breast Cancer. 2019 Dec;19(6): e683-89.

35 Chow LW, Yip AY, Loo WT, Lam CK, Toi M. Celecoxib anti-aromatase neoadjuvant (CAAN) trial for locally advanced breast cancer. J Steroid Biochem Mol Biol. 2008 Jul; 111(1-2):13-7.

36 Haddad TC, Goetz MP. Landscape of neoadjuvant therapy for breast cancer. Ann Surg Oncol. 2015 May;22(5):1408-15.

37 Dowsett M, Smith IE, Ebbs SR, Dixon JM, Skene A, Griffith C, et al. Proliferation and apoptosis as markers of benefit in neoadjuvant endocrine therapy of breast cancer. Clin Cancer Res. 2006 Feb;12(3 Pt 2):1024s-30s.

38 Vatte C, Al Amri AM, Cyrus C, Chathoth S, Alsayyah A, Ahmad A, et al. Helical and kinase domain mutations of PIK3CA, and their association with hormone receptor expression in breast cancer. Oncol Lett. 2019 Sep; 18(3):2427-33
39 Martínez-Sáez O, Chic N, Pascual T, Adamo B, Vidal M, González-Farré B, et al. Frequency and spectrum of PIK3CA somatic mutations in breast cancer. Breast Cancer Res. 2020 May;22(1):45

40 O'Leary B, Hrebien S, Morden JP, Beaney M, Fribbens C, Huang X, et al. Early circulating tumor DNA dynamics and clonal selection with palbociclib and fulvestrant for breast cancer. Nat Commun. 2018 Mar;9(1):896.

41 Finn R, Jiang Y, Rugo H, Moulder SL, Im SA, Gelmon KA, et al. Biomarker analyses from the phase 3 PALOMA-2 trial of palbociclib (P) with letrozole (L) compared with placebo (PLB) plus L in postmenopausal women with $\mathrm{ER}+$ /HER2- advanced breast cancer (ABC). Ann Oncol. 2016 Oct;27(6):1-36.

42 Dowsett M, Smith IE, Ebbs SR, Dixon JM, Skene A, A'Hern R, et al. Prognostic value of Ki67 expression after short-term presurgical endocrine therapy for primary breast cancer. J Natl Cancer Inst. 2007 Jul;99(2):167-70.

43 Baselga J, Semiglazov V, van Dam P, Manikhas A, Bellet M, Mayordomo J, et al. Phase II randomized study of neoadjuvant everolimus plus letrozole compared with placebo plus letrozole in patients with estrogen receptor-positive breast cancer. J Clin Oncol. 2009 Jun;27(16):2630-7.

44 Chow LWC, Morita S, Chow CYC, Ng W-K, Toi M. Neoadjuvant palbociclib on ER plus breast cancer (N007): clinical response and EndoPredict's value. Endocr Relat Cancer. 2018 Feb;25(2):123-30. 